\title{
$\alpha$-fetoprotein-producing endometrial adenocarcinoma without an obvious hepatoid component
}

\author{
JUNICHI KODAMA ${ }^{1}$, NORIKO SEKI ${ }^{1}$, HIROYUKI YANAI ${ }^{2}$, TOMOYUKI KUSUMOTO ${ }^{1}$, \\ KEIICHIRO NAKAMURA ${ }^{1}$, ATSUSHI HONGO ${ }^{1}$ and YUJI HIRAMATSU ${ }^{1}$
}

\author{
${ }^{1}$ Department of Obstetrics and Gynecology, Okayama University Graduate School of Medicine, Dentistry and \\ Pharmaceutical Sciences; ${ }^{2}$ Department of Pathology, Okayama University Hospital, Okayama 700-8558, Japan
}

Received July 31, 2009; Accepted September 21, 2009

DOI: 10.3892/ol_00000043

\begin{abstract}
Endometrial carcinomas producing $\alpha$-fetoprotein (AFP) are extremely rare. We report a case of AFP-producing, Grade 2 endometrioid adenocarcinoma without an obvious hepatoid component. A 59-year-old multiparous woman presented with abdominal swelling. The serum level of AFP was $1292.8 \mathrm{ng} / \mathrm{ml}$. Magnetic resonance images of the pelvis showed a markedly enlarged uterus, with a tumor growing exophytically within the endometrial cavity. Total abdominal hysterectomy and bilateral salpingo-oophorectomy were performed. The histopathological examination demonstrated a Stage IIA, Grade 2 endometrioid adenocarcinoma without an obvious hepatoid component. The endometrioid adenocarcinoma cells were partly positive for AFP immunoreactivity. The patient received adjuvant chemotherapy and was diseasefree 60 months after the operation. This case confirms the existence of AFP-producing endometrioid adenocarcinomas without hepatoid features.
\end{abstract}

\section{Introduction}

$\alpha$-fetoprotein (AFP) is a significant serum protein synthesized during fetal life, and the expression of the AFP gene is dramatically reduced after birth. Numerous cases of AFP-producing carcinomas have been reported, with stomach as the most common site (1). It has been demonstrated that AFP-producing gastric cancers are clinically aggressive and have a poor prognosis (2). However, AFP-producing endometrial tumors, with the exception of yolk sac tumors, are extremely rare, and only 12 cases of the former have been described in the English literature (3-14). In such cases, the serum level of AFP is generally elevated and AFP production can be demonstrated by immunohistochemical analysis of the

Correspondence to: Dr Junichi Kodama, Department of Obstetrics and Gynecology, Okayama University Graduate School of Medicine, Dentistry and Pharmaceutical Sciences, 2-5-1 Shikata-cho, Kita-ku Okayama 700-8558, Japan

E-mail: kodama@cc.okayama-u.ac.jp

Key words: endometrial adenocarcinoma, $\alpha$-fetoprotein, hepatoid cytoplasm, especially in hepatoid adenocarcinoma (HC) cells. We report on a case of AFP-producing, Grade 2 endometrioid adenocarcinoma without an obvious hepatoid component.

\section{Case report}

The patient was a 59-year-old (gravida 2, para 2) Japanese woman. Except for diabetes mellitus since the age of 57, her family and past medical history were routine. Age of menopause was 50 years. The patient presented at a local clinic complaining of abdominal swelling that had persisted for a few months. Serum AFP levels were elevated and a pelvic tumor was detected. The patient was referred to our hospital. No vaginal bleeding nor palpable lymphadenopathy were detected. A pelvic examination showed a tumor approximately the same size as a 16 -week gestational uterus. No abnormalities were observed in the indices of the complete blood count and serological tests. Serum levels of AFP and CA-125 were $1292.8 \mathrm{ng} / \mathrm{ml}$ (normal $<10 \mathrm{ng} / \mathrm{ml}$ ) and $82.0 \mathrm{U} / \mathrm{ml}$ (normal $<35 \mathrm{U} / \mathrm{ml}$ ), respectively, while those of other tumor markers including CA 19-9, CA 72-4 and CEA were within the normal range. Magnetic resonance imaging of the pelvis showed a markedly enlarged uterus, with a tumor growing exophytically within the endometrial cavity along with fluid collection and an adnexal tumor. The uterine tumor was heterogeneously hypointense with scattered areas of slightly high signal intensity on T2-weighted imaging (Fig. 1A) and appeared hypointense to the myometrium on contrastenhanced T1-weighted imaging (Fig. 1B). Computerized tomography showed no signs of lymph node, peritoneal, lung or liver metastases.

Abdominal exploration revealed a markedly enlarged uterus, and a left ovarian cyst and hydrosalpinx adhesive to the posterior wall of the uterine corpus. A small amount of ascites was present and the cytological examination was negative for malignant cells. No dissemination was observed in the peritoneal cavity. Total abdominal hysterectomy, bilateral salpingo-oophorectomy and partial omentectomy were carried out. Lymphadenectomy was not performed, since the adhesions in the pelvic cavity were extremely strong. The uterus along with the left adnexal tumor weighed $1,850 \mathrm{~g}$. A histopathological examination showed a Grade 2 endometrioid adenocarcinoma invading more than half of the thickness of the myometrium, extending into the cervical mucosa, with 

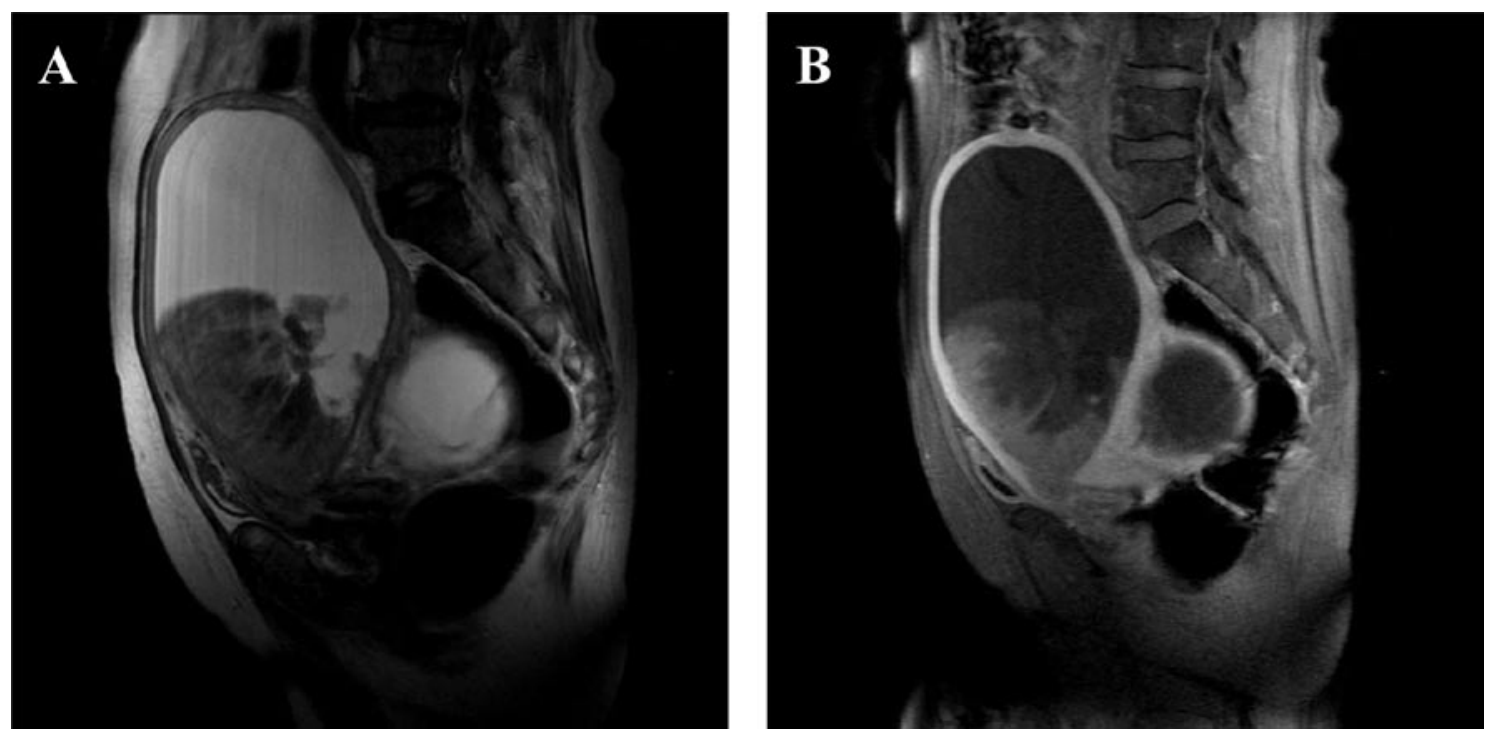

Figure 1. Magnetic resonance (MR) images showing a tumor growing exophytically within the endometrial cavity and fluid collection in the uterine cavity. (A) Sagittal T2-weighted image. (B) Sagittal contrast-enhanced T1-weighted image.
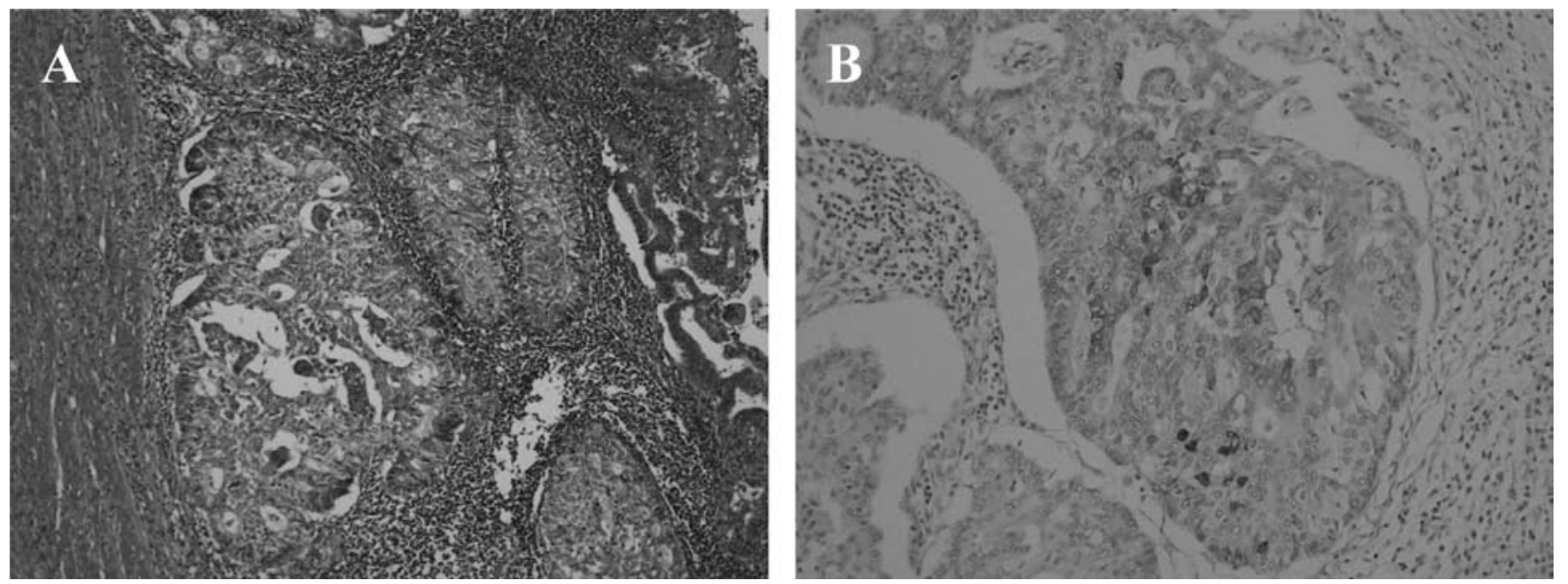

Figure 2. (A) Photomicrograph of uterine corpus showing Grade 2 endometrioid adenocarcinoma. (B) Immunohistochemical staining of AFP showing intracytoplasmic localization in endometrioid adenocarcinomas cells.

lymphatic invasion and no evidence of malignancy in the left adnexal tumor (Fig. 2A). Hepatoid adenocarcinoma cells were not detected in any of the tissue sections obtained. The endometrioid adenocarcinoma cells were partially immunoreactive for AFP (Fig. 2B). The disease was consistent with Stage IIA endometrial cancer, according to the International Federation of Gynecology and Obstetrics (FIGO) classification system. The patient was intravenously administered six courses of a multidrug regimen consisting of carboplatin, therarubicin and paclitaxel. Levels of serum AFP declined to the normal range on the 42nd postoperative day. Drug therapy was welltolerated and the patient has remained disease-free 60 months after the operation.

\section{Discussion}

$\mathrm{HC}$ is a variant of adenocarcinoma that is composed of tumor cells with extensive hepatic differentiation. The first case of $\mathrm{HC}$ was reported in 1985 as an AFP-producing gastric carcinoma with features of hepatic differentiation (15). HC usually generates an expanding mass with scattered or extensive necrosis. Numerous cases of carcinomas with hepatoid differentiation were reported in a variety of primary organs, including the gastrointestinal tract, ovary, pancreas, lung, kidney, urinary bladder and uterus (1). HC should essentially be diagnosed on the basis of the histological features of the tumor. It is characterized by the medullary or papillotubular arrangement of tumor cells with eosiophilic and granular cytoplasm, thus resembling hepatocellular carcinoma.

In 13 reported cases of AFP-producing endometrial carcinomas, including the present case, the patients were elderly (median 63 years; range 44-68 years) (3-14). These tumors usually show an exophytic growth pattern and are histologically high-grade. Furthermore, patients are diagnosed at advanced stages and have poor prognosis. AFP expression has been demonstrated in $\mathrm{HC}$ cells. However, previously published reports, did not address AFP expression in HCs $(4,5)$. In the 
cases of carcinosarcoma, AFP was reported to be detected in the carcinomatous but not in the sarcomatous components (3).

The present case was unusual in comparison with most reported cases in that the tumor was not high-grade, and hepatoid features were not detected in any of its sections. Yamamoto et al reported that AFP expression was detected in endometrioid adenocarcinoma cells and was particularly strong in the hepatoid cells (7). The relationship between endometrioid adenocarcinoma and hepatoid cells is completely unknown. In our case, the aberrant differentiation of adenocarcinoma cells into cells with phenotypes of hepatocytes may be weak. Toyoda et al reported that it is difficult to determine whether an endometrial tumor produces AFP solely on the basis of the morphological features of the tumor (9). Nagai et al reported cases of $\mathrm{HCs}$ and AFP-positive adenocarcinomas without heparoid features (APC) of the stomach (16). The incidence of venous invasion in $\mathrm{HC}$ was higher than that of APC. There were no significant differences between the two tumors regarding clinical features, macroscopic features and the incidence of lymphatic permeation. As for advanced carcinomas, the prognosis of patients with $\mathrm{HC}$ was poorer than that of patients with APC. Therefore, previous investigators stated that HC should be distinguished from APC. Our case suggests the occurrence of endometrial AFP-positive adenocarcinomas without hepatoid features.

Serum AFP does is not commonly examined in cases of endometrial cancer. Therefore, the true frequency of endometrial AFP-producing adenocarcinomas without obvious hepatoid features remains unclear. The endometrium is a possible site of AFP-producing carcinomas and further studies are essential to clarify this issue.

\section{References}

1. Kishimoto T, Nagai Y, Kato K, Ozaki D and Ishikura H: Hepatoid adenocarcinoma: a new clinicopathological entity and the hypotheses on carcinogenesis. Med Electron Microsc 33: 57-63, 2000.

2. Adachi Y, Tsuchidashi J, Shiraishi N, Yasuda K, Etoh T and Kitao S: AFP-producing gastric carcinoma: multivariate analysis of prognostic factors in 270 patients. Oncology 65: 95-101, 2003.
3. Kawagoe K: A case of mixed mesodermal tumor of the uterus with alpha-fetoprotein production. Jpn J Clin Oncol 15: 577-583, 1985.

4. Matsukuma K and Tsukamoto N: Alpha-fetoprotein producing endometrial adenocarcinoma: report of a case. Gynecol Oncol 29: 370-377, 1988.

5. Kubo K, Lee GH, Yamauchi $\mathrm{K}$ and Kitagawa T: Alphafetoprotein-producing papillary adenocarcinomas originating from a uterine body. A case report. Acta Pathol Jpn 41: 399-403, 1991.

6. Hoshida Y, Nagakawa T, Mano S, Taguchi K and Aozasa K: Hepatoid adenocarcinoma of the endometrium associated with alpha-fetoprotein production. Int J Gynecol Pathol 15: 266-269, 1996.

7. Yamamoto R, Ishikawa $H$, Azuma M, Hareyama $H$, Makinoda $S$, Koyama Y, Nishi S and Fujimoto S: Alpha-fetoprotein production by a hepatoid adenocarcinomas of the uterus. J Clin Pathol 49: 422-425, 1996.

8. Phillips KA, Scurry JP and Toner G: Alpha-fetoprotein production by a malignant mixed mullerian tumor of the uterus. J Clin pathol 49: 349-351, 1996.

9. Toyoda H, Hirai T and Ishii E: Alpha-fetoprotein producing uterine corpus carcinoma: a hepatoid adenocarcinomas of the endometrium. Pathol Int 50: 847-852, 2000.

10. Adams SF, Yamada D, Montag A and Rotmensch R: An alpha-fetoprotein-producing hepatoid adenocarcinoma of the endometrium. Gynecol Oncol 83: 418-421, 2001.

11. Takano M, Shibasaki T, Sato K, Aida S and Kikuchi Y: Malignant mixed mullerian tumor of the uterine corpus with alpha-fetoprotein-producing hepatoid adenocarcinoma component. Gynecol Oncol 91: 444-448, 2003.

12. Takahashi $\mathrm{Y}$ and Inoue $\mathrm{T}$ : Hepatoid carcinoma of the uterus that collided with carcinosarcoma. Pathol Int 53: 323-326, 2003.

13. Takeuchi K, Kitazawa S, Hamanishi S, Inagaki M and Murata $\mathrm{K}$ : A case of alpha-fetoprotein-producing adenocarcinoma of the endometrium with a hepatoid component as a potential source for alpha-fetoprotein in a postmenopausal woman. Int J Gynecol Cancer 16: 1442-1445, 2006.

14. Tran TA, Ortiz HB, Holloway RW, Bigsby GE and Finkler NJ: Alpha-fetoprotein-producing serous carcinoma of the uterus metastasizing to the ovaries, mimicking primary ovarian yolk sac tumor: a case report and review of the literature. Int J Gynecol Pathol 26: 68-70, 2007.

15. Ishikura H, Fukasawa $Y$, Ogasawara K, Natori T, Tsukada $Y$ and Aizawa M: An AFP-producing gastric carcinoma with features of hepatic differentiation: case report. Cancer 56: 840-848, 1985.

16. Nagai E, Ueyama $T$, Yao $T$ and Tsuneyoshi M: Hepatoid adenocarcinomas of the stomach. A clinicopathological and immunohistochemical analysis. Cancer 72: 1827-1835, 1993. 